\title{
Bimodal Spatial and Temporal Influences on Working Memory Performance
}

Ghazi, T.R. ${ }^{1}$ and Courtney, S.M. ${ }^{1,2}$

${ }^{1}$ Department of Psychological and Brain Sciences, Johns Hopkins University, Baltimore, MD, 21218, USA

${ }^{2}$ Department of Neuroscience, Johns Hopkins University, MD 21205, USA

Corresponding Author:

Tara R. Ghazi

Email: tararghazi@gmail.com

Declarations of interest: none 


\section{Introduction}

The environments we exist within offer up rich and ongoing sensory information, yet we typically pay attention to and make use of only a small subset of that information. Selective attention has a critical role in working memory processes, which we rely upon to achieve goaldriven tasks (van Ede \& Nobre, 2021). What we attend to, therefore, can have a large impact on the outcome of our performance. Where we allocate our attention, matters. In a busy city filled with talking people and rumbling vehicles, perhaps sounds coming from our left and right matters most in our determining whether to step out into the street. In a wooded forest with squirrels bounding over dry leaves and snakes creating a rustle as they carve out paths over the ground, if we are hoping to spot a rare bird perhaps only sounds from above will catch our interest. In a quiet library, where the turning of pages or clicking of fingers on a keyboard pose small violations to an otherwise silent atmosphere, if our goal is to remain focused on our studies, we may try to shift attention away from any sound in an effort to do so. During those moments in which our attention is focused and our working memory engaged, when both sights and sounds are available and potentially relevant, what information do we utilize? And to what effect on our performance? Herein we explore these questions as they relate to performance in a visuospatial working memory task when spatially parameterized stimuli in both visual and auditory modalities are present.

Sounds and sights may each provide us with potentially task-relevant information, though via different sensory modality streams. In cases where both modality streams seem to be reinforcing the same relevant information, it may be advantageous to integrate the two. For example, if there are cues available to us that different streams originate from the same singular source, by way of multimodal integration we may conceptually bind information from those 
streams together (Talsma et al., 2010). Multisensory inputs that share temporal and spatial features, in particular, are likely to be integrated and processed as singular unified events (Welch, 1999).

This type of multimodal binding occurs automatically and without conscious effort — we do not have to think about it for it to happen. In part, this is because the brain has classes of neurons which are specialized for the rapid computation and processing of conjunctive events. Some multisensory neurons in superior colliculus, for example, are tuned to respond to temporal conjunctions between input from different modalities (Meredith et al., 1987). Cellular networks within the cerebellum, support discrimination between temporal occurrences (Ivry \& Spencer, 2004). In primary sensory cortices, there exist unimodal and crossmodal neurons which become stimulated when neurons in other sensory regions are activated. These cells promote amplified responses to input in their native sensory modality when those inputs occur in temporal conjunction with inputs from other modalities (Allman \& Meredith, 2007; Allman et al., 2008). At the population level, neuronal activity in primary auditory cortex can have a modulatory effect on activity in visual cortex (Mercier et al., 2013). These circuits of activity have been directly linked to facilitation of behavior (Stein et al., 1989).

Note that the terms "multimodal" and "bimodal" are used to indicate the presence of stimuli in different sensory modalities. At times, bimodal stimuli - such as a sight and a sound - may be attributed, correctly, to a singular source. At other times, however, bimodal stimuli may arise from difference sources. While the brain is well adapted to rapidly bind discrete percepts together, to facilitate comprehension of them (Talsma et al., 2010), the stimuli themselves exist separately from any unifying representations we may form.

Past studies have found that when visual and auditory stimuli are available to us, the combination can benefit behavioral performance in visual tasks. A simple sound "pip" presented 
along with a busy visual scene, for example, can speed up a visual search for a target (Van der Burg et al., 2008). An oddball sound, embedded in a stream and presented in synchrony with a visual array can speed up visual detection (Vroomen \& De Gelder, 2000). People are faster to detect location changes of bimodal visual and auditory stimuli, than of stimuli in either modality alone (Schröger \& Widmann, 1998). At other times, however, the presence of multimodal streams can lead to errors. A single visual stimulus accompanied by a rapid succession of discrete sounds, for example, can generate the false perception of multiple visual stimuli despite the presentation of only one (Mishra et al., 2007; Shams et al., 2000). When participants must determine a direction of stimulus movement, if both visual and auditory stimuli are presented together, moving in opposite directions, the conflicting presentation has a negative impact on performance. Interestingly, however, it is only the case that performance is worse when reporting the direction of sound presented with conflicting visual information, but not vice-versa (Soto-Faraco et al., 2004). Errors in word perception can occur when the sound of words and the mouth movements of a speaker are in conflict (McGurk \& Macdonald, 1976). Engaging in a separate visual task while still viewing a speaker and listening to mismatches, though, eliminates these errors (Alsius et al., 2005).

The presence of sound may have different effects on performance in a visual task dependent upon either the demands of the task or the features of the sound. In a study by Marsh et al., for example, participants were presented with a visual sequence of seven Navon letters to encode into verbal working memory while an irrelevant stream of sounds was simultaneously presented. When the focal demands of the task were increased - described in this study as high working memory load - performance suffered far less from the presence of an auditory stream than when working memory load was low. In a follow-up experiment, however, participants were exposed to a sound 
stream that continually changed while trying to encode a visually presented verbal sequence. The presence of this dynamically changing sound had a negative impact on performance regardless of working memory load (Marsh et al., 2020).

We have some degree of control over what information we prioritize and selectively attend to among multiple modality streams. Over time, repetitive and irrelevant sounds can be endogenously suppressed, for example, reducing the amount of attention directed to them (Todorovic et al., 2011). However, we are never able to flip a cognitive switch that can selectively cut off input from one modality entirely (Lorenc et al., 2021; Myers et al., 2017). Simple, irrelevant sounds that temporally co-occur with a visual target can alter subjective visual perception (Barry E. Stein et al., 1996). Covert shifts of attention can be induced by auditory stimuli in modalities we are not actively or purposefully attending to (Driver \& Spence, 1998). In these examples, unrelated information arising from separate modality streams can have crossmodal effects that arise from their juxtaposition. If one is performing a visuospatial task in which visual and auditory stimuli co-occur in synchrony, then, information from the auditory modality, despite being irrelevant, may indeed impact performance.

Seemingly automatic integration effects, however, can be modulated by attentional strategies. If bimodal streams co-occur but we deem them independent of one another based on conceptual or contextual knowledge, there are different ways in which we might process the information arising from each. For instance, if both streams might be independently relevant, we might shift attention between the two modality streams. Doing so, however, may lead to slowing of our responses, or to errors when the streams contain conflicting information (Lukas et al., 2010). Another approach we might take is to actively focus on one modality while passively experiencing or trying to suppress the other. With this approach, however, we may miss potentially helpful 
information from the unattended stream (Gherri \& Eimer, 2011), or make errors as a result of conflicting information seeping through (Marsh et al., 2015). Errors of this latter form are just one example of an outcome resulting from intersensory bias - posited to be a means of reconciling discrepant perceptual information (Welch, 1999; Welch \& Warren, 1980). In studies where there are discrepancies in locational information between visual and auditory streams, a bias toward the location derived from the visual content has often been found (reviewed in Welch \& Warren, 1980). Visual dominance has also been found in a number of studies in which stimuli are presented in singular instances, rather than in streams (Colavita, 1974; Diaconescu et al., 2013; Egeth \& Sager, 1977; Lukas et al., 2010).

It may also be the case that different people process bimodal streams of information differently. In a study of visuospatial working memory by Gmeindl \& Courtney (Gmeindl \& Courtney, 2011), for example, participants completed a within-subjects task contrasting performance in the presence versus absence of sound. In the sound condition, while participants performed a visuospatial task in which a serial sequence of targets was presented, discrete noise bursts were presented in synchrony with the onset of each new visual target. Further, the spatial presentation of each noise burst was randomized such that the sound was either central, or completely or partially lateralized, in its presentation. Different results were found for different participants: in neurotypical individuals, the presence of the auditory stream indeed resulted in worse performance compared to silence - the spatially random sound was distracting. For individuals with a clinical multiple sclerosis diagnosis, however, performance was actually facilitated in the sound condition compared to when no sound was present (Gmeindl \& Courtney, 2011). 
If immersed in a primarily visual task, we might consider all sounds to be a distraction as in the case of unexpected noises occurring while studying in a library. But yet in some instances, and for some individuals, even potentially conflicting sounds may be helpful. If an event does indeed negatively impact or interfere with performance, objectively it can be classified as "distracting." A sound causing distraction can typically be attributed to one of two things: either the sound pulled attentional resources away from where they were previously allocated, or it interfered with ongoing task-related processing of information (Hughes, 2014). Trying to remember an ongoing stream of visual information in the presence of an auditory stream that is continually changing, as in Gmeindl \& Courtney (2011), is an example of the latter. As demonstrated in that study, however, distraction is not always clearly predictable.

Many studies that have informed our understanding of the behavioral impact of bimodal visual and auditory stimulus presentation have focused on effects of perception or attention in particular (Egeth \& Sager, 1977; Van der Burg et al., 2008; Villena-González et al., 2016). In studies examining the impact of bimodal stimulus streams upon working memory, the effects upon memory for verbal information in particular have been well studied (Hughes et al., 2013; Hughes \& Marsh, 2019; Marsh et al., 2015). The effects of bimodal presentation upon working memory for visuospatial information, however, have been far less examined. Within the described visuospatial working memory study that employed spatially random auditory distractors (Gmeindl \& Courtney, 2011), distraction, rather than bimodal integration or interference in particular, was the subject of focus. The question, therefore, of how bimodal visual and auditory streams may affect visuospatial working memory performance remains an open one.

Summarizing what has been discussed above, temporal coincidence between bimodal stimuli in particular can encourage either multimodal binding or integration (Talsma et al., 2010). 
Further, if bimodal stimuli share properties such as spatial location, information from both modalities may be relevant and improve performance on a spatial task. On the other hand, distraction and impaired working memory performance can arise due to effects of incongruency between a target and an unrelated stimulus (Diaconescu et al., 2011, 2013; Lorenc et al., 2021). Studies that present visual and auditory stimulus streams concurrently have demonstrated that when task goals warrant attending primarily to the visual stream, an auditory stream that is changing unpredictably in space is likely to capture attention and impact performance (Gmeindl \& Courtney, 2011; Marsh et al., 2020; Welch, 1999). But yet, many studies have found that for processing of spatial information in particular, vision is the dominant sense (Diaconescu et al., 2013; Egeth \& Sager, 1977; Lukas et al., 2010). Further, processing of repetitive auditory stimuli with constant parameters (such as tone) and predictable timing, can be suppressed (Todorovic et al., 2011). Thus, it remains unclear under what circumstances irrelevant and potentially conflicting auditory stimuli will or will not interfere with working memory for spatial information in the visual modality.

We hypothesized that streams of sound providing spatial information will be integrated in working memory during performance of a visuospatial task, but only when those sounds temporally co-occur with visual targets. Further, we hypothesized that this synchrony-induced integration will have differential effects on performance dependent upon spatial congruence. We predicted auditory stimuli that temporally co-occur with visual stimuli will facilitate performance when the spatial information provided by both are congruent, but hinder performance when the spatial information of each is incongruent, and therefore in conflict. 


\section{Methods}

To test these hypotheses, we adapted the working memory paradigm utilized in Gmeindl \& Courtney (2011). The task in this study was itself a modified version of the Corsi Blocks paradigm (Corsi, 1972), which has been employed for decades in the assessment of an individual's visuospatial working memory span (Berch et al., 1998; Monaco et al., 2013). Generally, in this paradigm, a sequence of squares ('blocks') are selected from an array and presented as memory targets, with one target square identified at a time. Participants must remember every target presented and reproduce the sequence following a brief delay.

This visuospatial working memory task is described in detail below. As in Gmeindl \& Courtney (2011), spatial manipulations of sound stimuli were achieved by lateralizing their presentation using interaural intensity differences with headphones.

\subsection{Participants}

Young adult participants were recruited from the Johns Hopkins University student community for participation and were compensated via course credit. Participants ranged in age between 18 and 28 years, and included 30 females (mean age $=19.8, \mathrm{SD}=1.20$ ) and 30 males (mean age $=19.9, \mathrm{SD}=1.98)$, categorized based upon binary self-report of gender. Participants were screened via self-report for typical hearing in both ears, normal or corrected-to-normal vision, and lack of attention or memory related clinical diagnoses or traumatic brain injury.

\subsection{Stimuli and Task}

Auditory stimuli

A single auditory stimulus was utilized for the task. This sound stimulus was a $300 \mathrm{~ms}$ segment of pink noise, with a 10ms rise and fall at onset and offset, respectively. Pink noise, unlike white noise, has a decaying power frequency spectrum and therefore is akin to naturally occurring 
sounds, without being generally associated with any specific semantic meaning. As pink noise has been shown to elicit a strong exogenous response (Wetzel et al., 2016), it was deemed an adequately salient, yet semantically neutral, sound stimulus with which to test our hypotheses. Two versions of this stimulus were created, one with $100 \%$ left-, the other $100 \%$ right-channel lateralization. Sounds were created in Audacity ${ }^{\circledR}$ sound editing software (Audacity Team, 1999). Auditory stimuli were presented through Sennheiser HD 201 over-ear binaural headphones, at a volume well above threshold.

\section{Visual Stimuli}

Visual stimuli consisted of 10 square "blocks" presented on a neutral gray colored background. Each block was dark gray in color and occupied approximately $3.6^{\circ}$ in visual angle of both height and width. Viewing distance was fixed at approximately $59 \mathrm{~cm}$ from the screen using a fixed-position chinrest. A configuration of five blocks was created wherein each block was separated by greater than $2.0^{\circ}$ of visual angle in all directions. This five-block configuration was repeated on the left and right sides of the visual space, with a horizontal separation of approximately $7.0^{\circ}$ of visual angle between them. On designation of a particular block as a target, the color of the block changed to cyan blue on the next screen refresh.

\section{Task}

In Figure 1 the on-screen configuration of visual stimuli is shown (A). For each trial a random sequence of six visual targets was selected from among the ten blocks within the visual array to be the Sample Sequence. A memory set size of six targets was determined to result in a suitable range of performance based upon pilot testing in this population. Targets were identified by an abrupt color change from grey to cyan blue, lasting $500 \mathrm{~ms}$. The visual inter-stimulus interval was $500 \mathrm{~ms}$, following which the next target in the sequence was presented. Following presentation 
of the entire Sample Sequence there was a 2.5 s delay before presentation of the Test Sequence. The Test Sequence was comprised of six visual targets that were either identical to, or different from, the Sample Sequence. On every trial, participants were to determine whether all targets within the Sample Sequence and Test Sequence matched in both location and order of presentation. Participants were cued to the onset of both the Sample Sequence and Test Sequence by a centrally presented fixation cross that lasted for a duration of $1 \mathrm{~s}$. Response was prompted by the display of "MATCH?" on screen until a keypress was made. No time limit was placed on responding. After a response was made, a 1s inter-trial interval preceded the start of the next trial. No feedback was provided.

During presentation of the Sample Sequence, on some trials an auditory stimulus (described above) was presented at the same time as each of the visual targets - with the onset of each occurring synchronously. In other trials, the onset of the auditory stimulus just preceded, or shortly followed, the onset of the visual stimulus. These trials are described as temporally asynchronous. Asynchronous auditory stimulus onsets randomly occurred either $100 \mathrm{~ms}, 200 \mathrm{~ms}$, or 300ms before, or after, onset of each visual target.

Synchronous and asynchronous trials were further defined by the spatial congruence between visual and auditory stimuli. In some trials, auditory stimuli were lateralized congruently with the spatial lateralization of the visual stimuli. These are described as congruent trials. In other trials, referred to as incongruent trials, the lateralization of each auditory stimulus was opposite of its corresponding visual stimulus in the sequence. These spatial relationships between auditory and visual stimuli are shown in section A of Figure 1.

During the Test Sequence, only visual stimuli were presented, with three possible Test Types. One third of the Test Sequences matched the Sample Sequence in both the identity of all 6 
targets and their order of presentation. One third of the Test Sequences mismatched the Sample Sequence in identity. In this Test Type a single target block was randomly selected from among those outside of the Sample Sequence and displayed in the third or fourth (randomly determined on each trial) position of the Test Sequence. The final third of Test Sequences were a mismatch in order. For mismatched order trials, the third and fourth targets within the Test Sequence were swapped in their ordinal positions. No instructions were provided to participants regarding trial types or auditory stimuli.

The task included a total of 75 trials. Sixty of the trials were bimodal, with both auditory and visual stimuli presented during the Sample Sequence. Fifteen trials were unimodal; only visual stimuli were presented. The 60 bimodal trials were divided among four conditions. As previously described, there were four Sample Conditions: Synchronous-Congruent, SynchronousIncongruent, Asynchronous-Congruent, and Asynchronous-Incongruent. Thus, there were 15 trials per Sample Condition, equally split among the three Test Types (5 Match, 5 Order-mismatch, and 5 Identity-mismatch trials).

The task was programmed in Python 3 (Python Software Foundation, 2008) utilizing the PsychoPy 3.2 (Peirce, 2007) library of code and software for delivery.

\subsection{Procedure}

After providing consent and receiving verbal and on-screen instructions and training, participants performed the task in a private booth seated in front of a computer screen with headphones on. For each participant, trials from each described Sample Condition were intermixed in a pseudorandomized order. Trials were separated into five experimental blocks with self-paced breaks permitted between blocks. On completion of the task, participants were given a 
demographics questionnaire and a then guided by the experimenter through a brief exit interview. The duration of the entire study session was approximately one hour.

\subsection{Design}

The study utilized a within-subjects, repeated-measures design. Randomized presentation of trials over the duration of the experiment, rather than a blocked design, was utilized to minimize habituation to any specific temporal or spatial occurrence of sound. Accuracy (proportion of correct responses) was the primary dependent variable of interest.

In bimodal trials, auditory and visual stimuli were presented only during the Sample Sequence to allow for study and interpretation of effects as they relate specifically to processes of working memory encoding. The Test Sequence always included only visual stimuli. Unimodal trials, in which only visual stimuli were presented during the Sample Sequence, provided a baseline with which to further compare and interpret results related to our hypotheses.

To test our hypotheses, parametric group-level comparisons were performed using omnibus ANOVA tests with Accuracy as the primary outcome variable. A 2x2 (Synchrony $\mathrm{x}$ Congruence) ANOVA test was performed to isolate the effects of different bimodal Sample Conditions. A $2 \times 2 \times 3$ (Synchrony $\mathrm{x}$ Congruence $\mathrm{x}$ Test Type) ANOVA test was performed to determine whether additional effects of Test Type were present and interacting with Sample Conditions. In cases where violations of sphericity were present, corrected statistics are reported. A 1-way ANOVA comparing all five Sample Conditions (4 bimodal, 1 unimodal) was performed. ANOVA tests were followed, when relevant, with paired $t$-tests to examine underlying effects. Multiple comparisons correction of $t$-test statistics was performed via the False Discovery Rate method (Benjamini \& Hochberg, 1995). 
Usage of a Yes/No recognition memory response procedure allowed for analysis of condition-specific responses using basic signal detection methods. Match trials in which participants responded "Yes" were marked as Hits. Mismatch trials in which participants responded "Yes" were marked False Alarms. Proportions of Hits and False Alarms were utilized to calculate $d$ ' as a measure of an individual's sensitivity to detect a match; $\beta$ their criterion in making a Yes/No decision (Green \& Swets, 1974).

All statistical analysis was performed in R (R Core Team, 2018) with the dplyr (Wickham et al., 2021), ez (Lawrence, 2016), and psycho (Makowski, 2018) packages. Figures were produced in R with the ggplot2 (Wickham, 2016), hrbrthemes (Rudis, 2018), and cowplot (Wilke et al., 2019) packages.

\section{Results}

Of the data collected, one participant was excluded from further analysis due to an attentional disorder and three due to failure to perform the task (below 60\% overall Accuracy). Data analyzed was from 56 participants (27 female, 29 male).

Our working hypotheses was that incongruence of spatial information between auditory and visual stimuli has a differential effect on spatial working memory dependent on whether sounds are synchronous with visual stimuli. A 2x2 (Congruence x Synchrony) omnibus ANOVA with Accuracy as the outcome variable resulted in a trending main effect of Congruence, $F(1,55)$

$=3.33, \mathrm{p}<0.075, \eta_{\mathrm{G}}^{2}<0.01$, and a significant interaction between Synchrony and Congruence,

$\mathrm{F}(1.55)=10.28, \mathrm{p}<0.005, \eta_{\mathrm{G}}^{2}=0.03$. This interaction can be seen in Figure 2. Follow-up paired 
$t$-tests were performed contrasting Accuracy between conditions. After correction for multiple comparisons, the difference in Accuracy between Synchronous-Congruent versus SynchronousIncongruent conditions was highly significant, $t=3.7, \mathrm{p}<0.002,95 \%$ C.I. [0.027,0.092]. That is, performance when bimodal stimuli synchronously co-occurred and exhibited spatial congruence was significantly greater than when stimuli synchronously co-occurred but were in spatial conflict. When sounds were presented asynchronously with the visual stimuli, the Accuracy difference between Congruent and Incongruent Conditions was not significant.

Comparison of Accuracy between Synchrony conditions when bimodal streams were spatially congruent was only a trending effect, $t=1.78, \mathrm{p}<0.08,95 \%$ C.I. [-0.004, 0.071]. For spatially congruent stimuli streams, temporal synchrony resulted in a marginal improvement of performance compared to temporal asynchrony. Alternatively, comparison of Accuracy between Synchrony conditions when bimodal streams were in spatial conflict remained significant after correction, $t=-2.46, \mathrm{p}<0.03,95 \%$ C.I. [-0.082, -0.008$]$. Performance was better, despite the spatial conflict, when bimodal streams were temporally asynchronous compared to synchronous. Temporal synchrony coupled with spatial conflict resulted in the worst performance. These results are shown in Figure 2.

A coarse comparison of visuospatial working memory performance in the context of bimodal (auditory and visual) versus unimodal (visual only) stimuli was performed by t-test between Accuracy in the unimodal condition versus Accuracy averaged across all bimodal conditions. No overall difference in performance was present, $t=1.13, \mathrm{p}>0.2$. A detailed comparison was performed via one-way ANOVA contrasting Accuracy across all conditions. The overall effect of Condition on Accuracy was significant: $F(4,220)=3.74, p<0.006, \eta_{G}^{2}=0.032$. 
Among the five Conditions, most relevant to our hypothesis were the direct contrasts of unimodal versus bimodal temporally synchronous conditions. Put otherwise - the comparison in performance of the visual task when no-sound versus synchronously occurring sound was present. These contrasts were performed via paired $t$-tests, corrected for multiple comparisons. Accuracy in the case of Synchronous-Incongruent bimodal stimuli was significantly worse than Accuracy for unimodal visual stimuli only, $t=-2.73, \mathrm{p}<0.02,95 \%$ C.I. [-0.078, -0.012$]$. Accuracy for Synchronous-Congruent bimodal stimuli, while numerically greater, was not statistically different from Accuracy given unimodal stimuli, $t=1.062, \mathrm{p}>0.2$.

Throughout the course of the experiment, participants were presented with trials of three different Test Types. The sequence presented during the Test period either matched the Sample Sequence or mismatched it in Identity or Order. To determine how Test Type contributed to the above effects on performance, a 2x2x3 (Synchrony x Congruence $\mathrm{x}$ Test Type) ANOVA was performed. In addition to the same effects of the previously reported 2x2 ANOVA, a main effect of Test Type, $\mathrm{F}(2,110)=38.06, \mathrm{p}<0.001, \eta_{\mathrm{G}}^{2}=0.16$, was present. A 3-way interaction did not exist. However, significant interactions between Congruence and Test Type, $F(2,110)=4.04, p<$ $0.03, \eta_{\mathrm{G}}^{2}<0.01$, and between Synchrony and Test Type, $\mathrm{F}(2,110)=4.19, \mathrm{p}<0.03, \eta_{\mathrm{G}}^{2}<0.01$, were present. These results are shown in Figure . Mean Accuracy is over 90\% across conditions for Identity-mismatch Test Type trials. Mean Accuracy for both Match and Order-mismatch Test Type trials ranged between $70-85 \%$. Again, the contrasts most relevant to our hypothesis were between the different spatial Congruence Conditions when stimuli were Synchronous. While the pattern of performance in Synchronous conditions was the same for both Match and Ordermismatch Test Types, the difference between Congruent and Incongruent spatial Conditions given 
an Order-mismatch Test Type was borderline marginal, $t=1.67, \mathrm{p}=0.1,95 \%$ C.I. [-0.011, 0.118]. The difference between these conditions given a Match Test Type, however, was large, $t=3.84, \mathrm{p}$ $<0.001,95 \%$ C.I. $[0.050,0.158]$.

Average sensitivity in detecting a Match, operationalized as $\boldsymbol{d}$ ', was computed and compared across Conditions. A significant difference in sensitivity was present based upon Condition: $F(4,220)=3.48, \mathrm{p}<0.01, \eta_{\mathrm{G}}^{2}=0.03$. Sensitivity was similar between Unimodal (visual stimuli only) and Bimodal Asynchronous Sample Condition trials. In Bimodal Synchronous trials, however, participants exhibited reduced sensitivity for spatially incongruent compared to congruent stimuli: $t=-3.81, \mathrm{p}<0.001,95 \%$ C.I. [-0.607, -0.188$]$.

The decision criterion, $\beta$, was found to significantly differ across Conditions, $F(4,220)=$ 3.12, $\mathrm{p}<0.02, \eta_{\mathrm{G}}^{2}=0.03$, but the effect is not driven by Conditions of Temporal Synchrony. Between the Synchronous conditions, there was little change in the average decision criterion, $\beta$, between spatial conditions, or compared to Unimodal visual-only stimuli: $t<1.0, \mathrm{p}>0.3$ for each comparison. Participants were not increasingly biased to respond Yes or No in the presence of synchronous sound. These results are shown in Figure .

\section{Discussion}

People utilize signals of timing and spatialization to selectively allocate attention and make inferences about the environment (Nobre \& van Ede, 2018; Theeuwes, 2014). These signals are sources of information. Studies of perception and attention have found that distinct signals arising from different sensory modalities can have a strong effect on behavior, particularly when those 
signals occur at the same time. In the present study, we present evidence that these effects extend to visuospatial working memory.

A significant difference in performance resulted when bimodal streams had synchronous compared to asynchronous temporal structure. We consider two possible ways by which information representation in working memory may have been affected, that account for this difference. First, by way of multimodal binding. Second, by way of reinforcement or interference.

Within synchronous trials, because auditory and visual information temporally coincided it may be the case that the two modality streams were representationally bound together (Talsma et al., 2010). For each target presented, then, the representation of that target's location would be formed based upon both visual and auditory information. In the case of spatially congruent bimodal streams, only accurate target location information would be available for representational binding, facilitating memory. In the case of spatially incongruent bimodal streams, only the visual stream would provide accurate location information while the auditory stream would be misleading. Binding auditory and visual streams in these instances would diminish the precision with which location was represented, hindering memory.

While this account does fit the resulting behavior observed, with Accuracy in task performance being greater for Synchronous-Congruent trials compared to SynchronousIncongruent trials, there are reasons this might be questioned. While both visual and auditory streams shared similarity in that they exhibited spatial and temporal structure, for example, they were not unified in any other obvious means - such as semantic connection. Pink noise, rather than an instrumental tone or an animal call was specifically utilized in this study for its lack of semantic meaning. Further, auditory stimuli were always presented through headphones. Previous studies have demonstrated that binding between auditory and visual stimuli is less likely when the two do 
not emanate from "out there" in space (Soto-Faraco et al., 2004). Another account then, is that bimodal streams were represented separately.

As reviewed in Chapter 2, decades ago it was demonstrated that people were able to remember and report fundamental features of sounds presented to them even when explicitly focusing their attention elsewhere (Treisman, 1964). In the present study, the spatial location of auditory stimuli may have been a sufficiently low-level feature for participants to perceive and encode, even if not explicitly directing attention to the auditory modality. When drawing upon memory during task performance, then, representations formed from visual stimuli may have been predominant, but auditory representations that had been separately formed and stored also had influence.

In considering the patterns of performance tied to different Test Types, of note is that the highest accuracy was achieved across conditions when sample and test target identities mismatched (see Figure ). Rather than separately encoding sample and test sequences, and then internally comparing representations between the two, reliance upon recognition memory is a far less demanding approach. An entirely mismatching target, then, would be readily identified as unfamiliar. In Match and Order-mismatch Test Types, performance was far lower.

Previous studies have demonstrated that visuospatial working memory in particular is more prone to errors of order in representing sequences of information than are other forms of working memory (e.g. Gmeindl et al., 2011). While the direction of difference between Accuracy in Synchronous-Congruent and Synchronous-Incongruent trials holds when it comes to Ordermismatch Test trials, the effect is marginal. There is a stronger effect of temporal structure in the case of Congruent trials, however, which is interesting. Performance is by far worst for trials in which bimodal stimuli were spatially congruent, but temporally asynchronous. Although the 
bimodal stimuli occurring in closest temporal proximity within Asynchronous-congruent trials were indeed spatially congruent, they may have been spatially incongruent relative to sequentially neighboring targets. Put otherwise, a single auditory stimulus in these trial types may have been spatially incongruent with an earlier, or later, visual stimulus. It is possible, then, that this effect may have arisen from proactive interference occurring across modalities. When auditory stimuli were asynchronous with visual stimuli, each auditory stimulus onset occurred randomly either before or after onset of each visual stimulus. In cases where the auditory stimulus was presented early, perceiving it may have generated interference in processes of consolidation related to the preceding visual target. In cases where the auditory stimulus was presented "late," it may have interfered with subsequent visual target encoding. When spatial incongruence was present with earlier or later targets, errors in memory for order may have resulted.

The largest Congruence-based difference within Synchronous trials arose within Match Test Type trials (Figure ). Indeed, in the case of Synchronous-Incongruent trials, participants exhibited the lowest sensitivity to detect a match (Figure 4A). As can be seen in Figure 4B, however, this was not due to a shift in decision Criterion to make a Yes/No response. Put otherwise, spatial conflict between synchronous bimodal stimuli did not lead participants to become more or less conservative in selecting a response than they were for Synchronous-Congruent or Asynchronous-Incongruent conditions. Only among Asynchronous-Congruent trials was there a comparatively marked shift in decision Criterion indicating a bias towards a Yes response.

As demonstrated by the behavioral differences across Sample Conditions and Test Types discussed, performance of a visuospatial working memory task was strongly influenced by the presence of information in the auditory modality. Synchronous inputs from different modalities may have generated some competition for attention. Even though visual dominance has been 
repeatedly demonstrated (e.g. Egeth \& Sager, 1977), there could at times be an outcome of competitive selection that favors dedicating neural resources to processing information arising from the auditory modality (Desimone \& Duncan, 1995; Knudsen, 2007). In the case of a dynamically changing auditory stream, change in the properties or features of specific sounds may lead to exogenous generation of bias and ultimately outcompete a visual stream for allocation of attention. On the other hand, endogenous signals, particularly those related to control processes, may win the resource "tug of war", keeping attention oriented in the most goal-appropriate direction. The sound stimulus used in the present study did not change within a trial, but the timing and lateralization of its presentation did. The impact was not so great as to drastically harm performance - Accuracy across Conditions was great than $70 \%$, but it clearly had an effect.

If the sound itself was consistently distracting, performance in Bimodal compared to Unimodal conditions should have suffered. This was not the case. Only when the sound was synchronous and spatially incongruent was there an effect of distraction - performance was worse in this case than in other Bimodal Conditions and worse than when no sound was present at all. Indeed $d$ ', sensitivity, was also lowest in this circumstance. This reinforces the similar finding when examining Accuracy by Test Type - given Synchronous and Incongruent sound, participants were least capable of distinguishing matches.

\section{Conclusion}

We hypothesized that the spatial information provided by bimodally presented visual and auditory stimuli would have the greatest impact on working memory performance when those stimuli occurred together in time. Indeed, this is exactly what we found. The effect, however, was not strictly beneficial. Instead, it differed based upon the congruence or incongruence of the spatial 
information provided by each sensory stream. While it may not be the case that information from each modality was integrated into a single representation, then stored in working memory, there is strong indication that representation arising from the perception of each modality did indeed impact performance. Temporal and spatial structure within, and across modalities, effect working memory representation. Future neuroimaging studies might shed light on the neural processing underlying this behavior. 


\section{Figures}

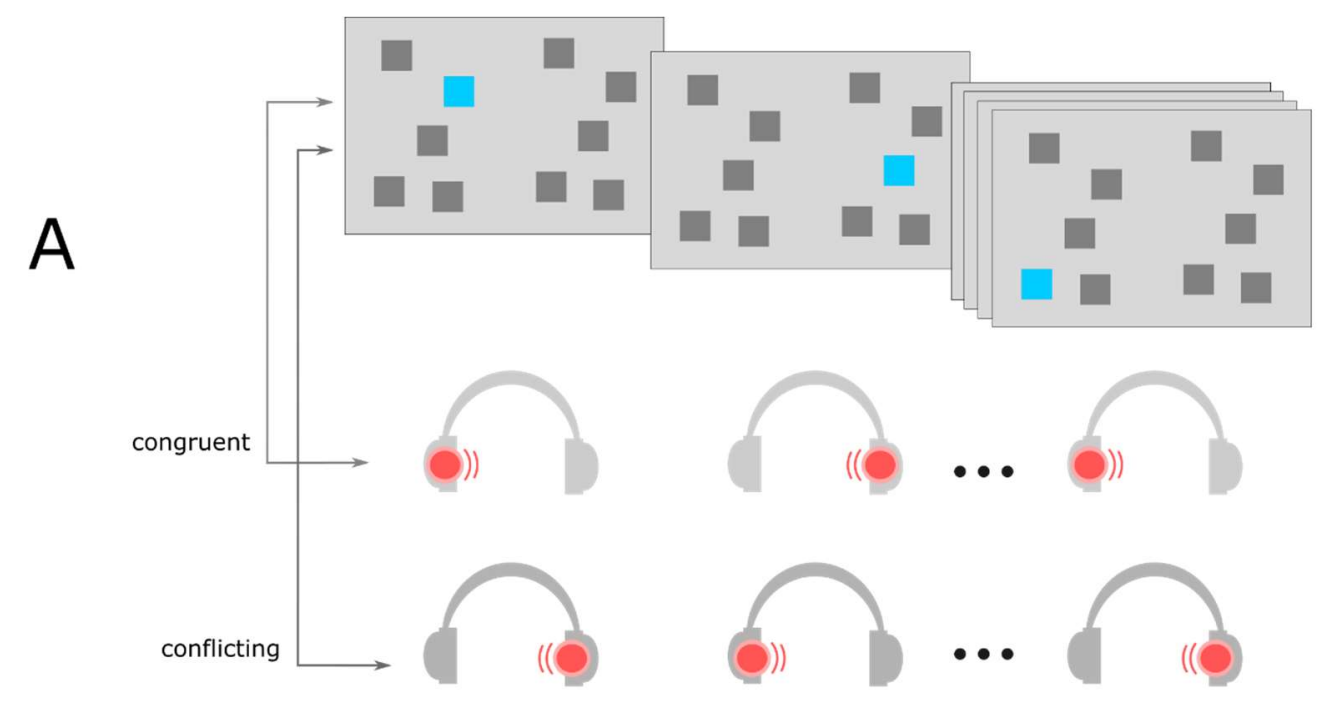

B

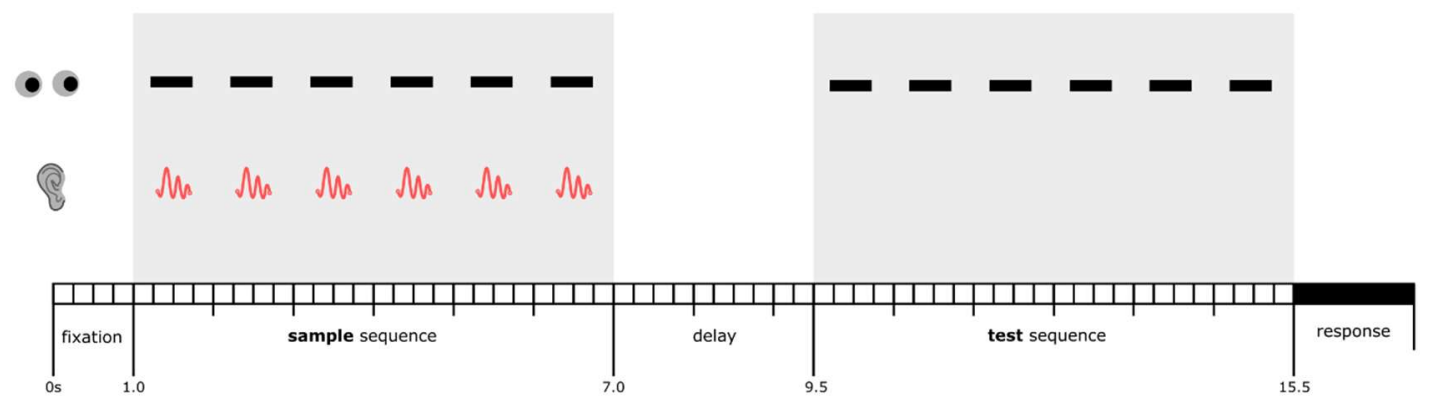

Figure 1: Overview of Task Conditions and Trial Timeline. A: Example of a Sample Sequence in which both visual and auditory stimulus streams are presented. Graphic depiction demonstrates the spatial relationship between visual targets (upper) and auditory (lower) stimuli in Congruent and Incongruent conditions. On every trial, six unique visual targets (cyan blue squares), selected at random, were presented. Ellipses and overlapping screens represent portions of the sequence presented during the experiment but not included in this visualization. B: Trial Timeline. Shaded rectangular regions indicate time periods of stimulus stream presentation. During presentation of the Sample Sequence, both visual and auditory stimulus streams were presented. During presentation of the Test Sequence, only visual stimuli were presented. In all task conditions, Participants were instructed to remember the Sample Sequence. After presentation of the Test Sequence, participants made a Yes or No response by keypress to indicate whether Sample and Test sequences matched. No instruction was given regarding the auditory stimuli. 


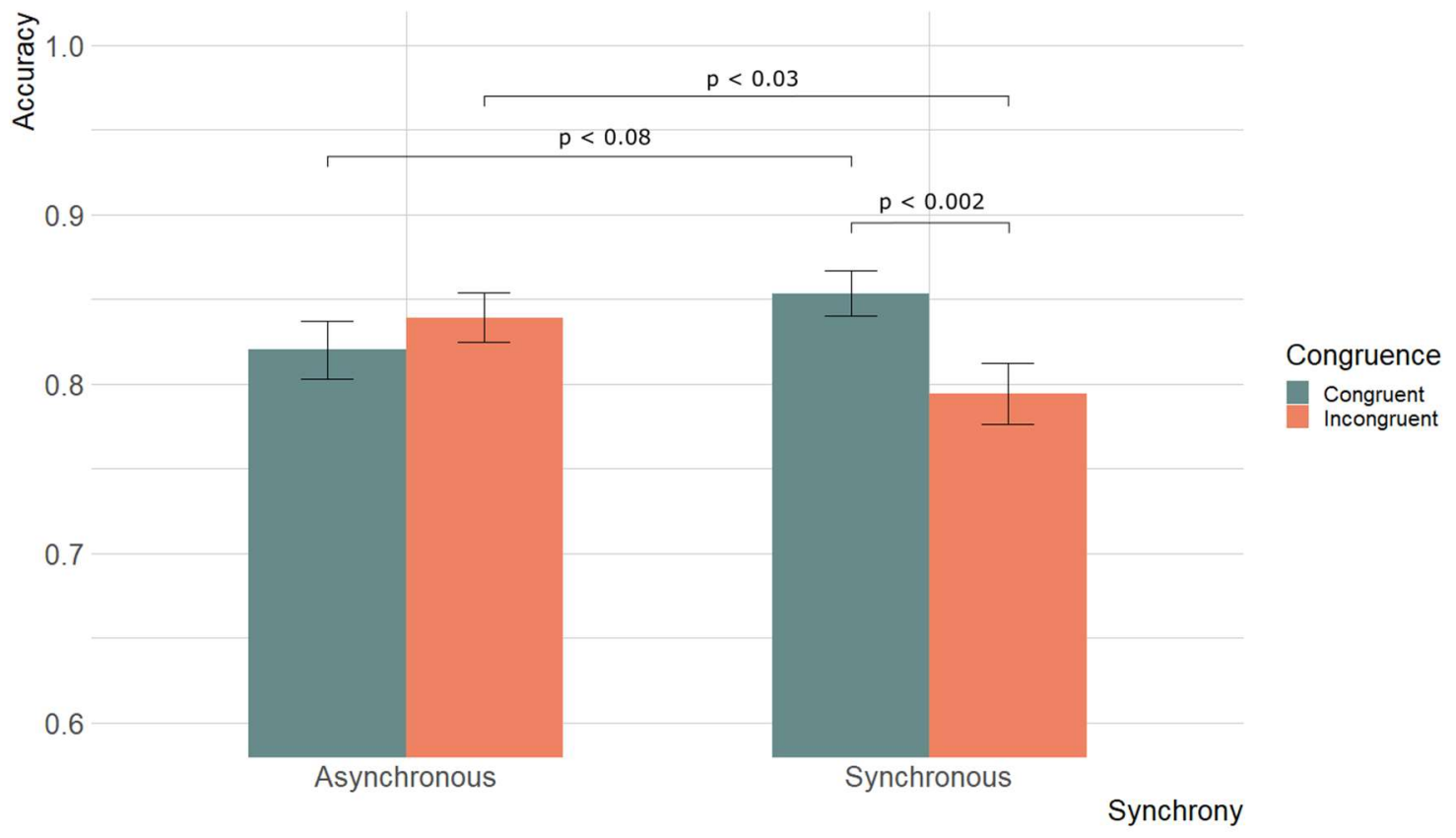

Figure 2: Accuracy (proportion correct) within bimodal trials by Sample Condition. Error bars indicate standard errors of the mean. Performance across Conditions in which bimodal stimuli were spatially Congruent are shown in deep turquoise; spatially Incongruent are shown in salmon. A significant interaction is present between Synchrony and Congruence, $\mathrm{F}(1.55)=10.28, \mathrm{p}<0.005$. Follow-up contrast statistics are shown. 


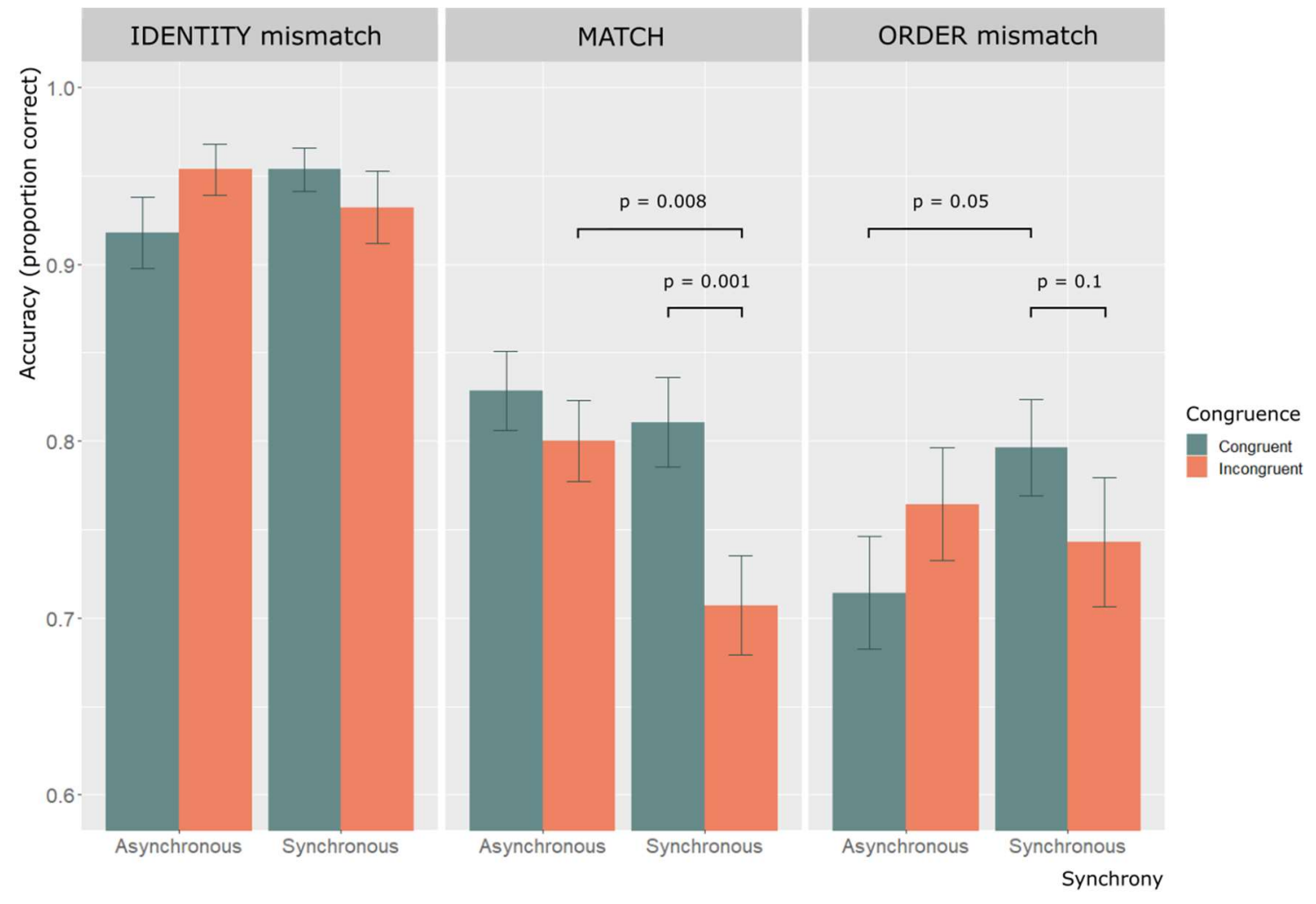

Figure 3: Performance (Accuracy) across bimodal trials by Sample Condition and Test Type. Trials were evenly split between the three Test Types. While the general pattern of Accuracy within Synchronous-Congruent trials being higher than Accuracy within Synchronous-Incongruent trials holds across Test Types, the difference between these conditions was greatest among trials in which the Test and Sample sequences matched. 
A

d' 2.1

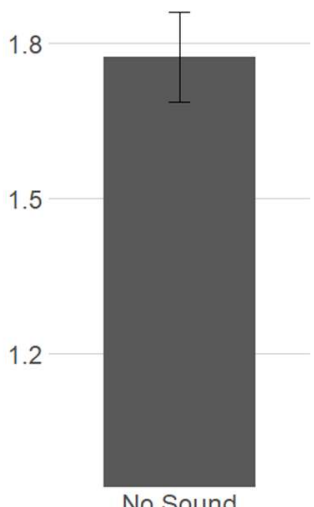

Unimodal d' 2.1

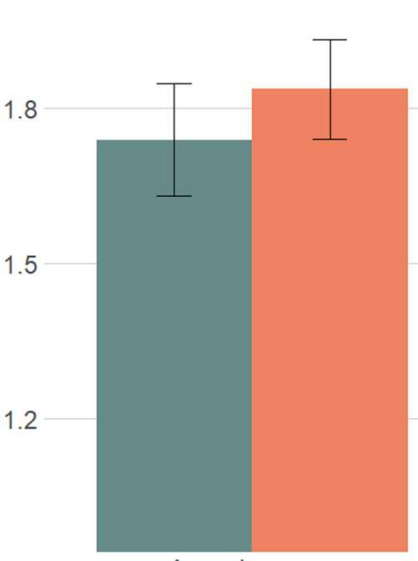

Asynchronous

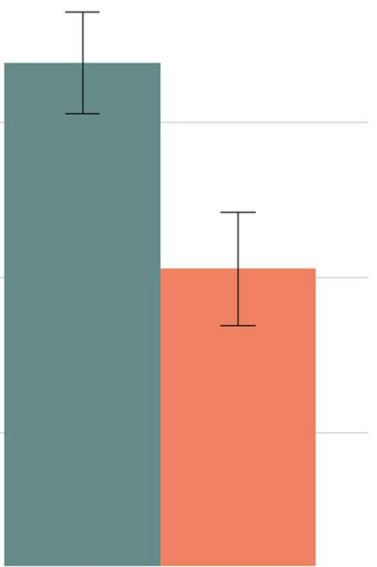

Synchronous

Bimodal
Congruence

Congruent

Incongruent

B

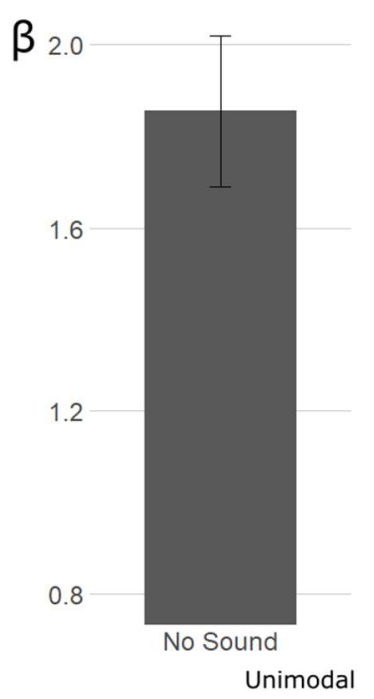

$\beta$

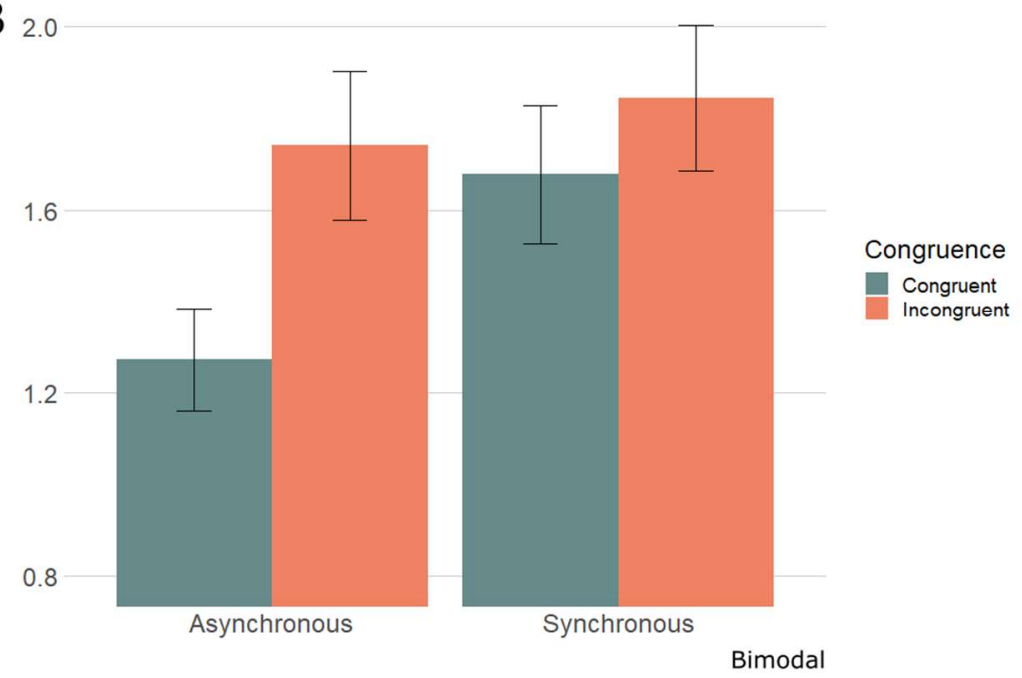

Figure 4: Comparison of participant sensitivity, d', (A) and decision criterion, $\beta,(\mathbf{B})$ across unimodal and bimodal Conditions. Main effects of Condition are present for both d' and $\beta$ outcome variables. The largest difference in sensitivity is present between the Synchronous-Congruent and Synchronous-Incongruent Bimodal conditions, indicating greater and lesser sensitivity, respectively, to detect Matching Sample and Test sequences. This reinforces the results shown in Figure. As shown in (B), however, this was not driven by a change in decision criterion these two conditions did not generate significantly more or less bias to make a Yes/No response. That $\beta$ was lowest for Asynchronous-Congruent trials indicates that participants were more biased to respond Yes, further shedding light on the differences in Accuracy by Test Type (Figure ) within this condition. 


\section{References}

Allman, B. L., \& Meredith, M. A. (2007). Multisensory Processing in "Unimodal" Neurons: Cross-Modal Subthreshold Auditory Effects in Cat Extrastriate Visual Cortex. Journal of Neurophysiology, 98(1), 545-549. https://doi.org/10.1152/jn.00173.2007

Allman, Brian L., Bittencourt-Navarrete, R. E., Keniston, L. P., Medina, A. E., Wang, M. Y., \& Meredith, M. A. (2008). Do cross-modal projections always result in multisensory integration? Cerebral Cortex, 18(9), 2066-2076. https://doi.org/10.1093/cercor/bhm230

Alsius, A., Navarra, J., Campbell, R., \& Soto-Faraco, S. (2005). Audiovisual integration of speech falters under high attention demands. Current Biology, 15(9), 839-843. https://doi.org/10.1016/j.cub.2005.03.046

Benjamini, Y., \& Hochberg, Y. (1995). Controlling the False Discovery Rate: A Practical and Powerful Approach to Multiple Testing. Journal of the Royal Statistical Society. Series B (Methodological), 57, 289-300. https://doi.org/10.2307/2346101

Berch, D. B., Krikorian, R., \& Huha, E. M. (1998). The Corsi block-tapping task: Methodological and theoretical considerations. Brain and Cognition, 38(3), 317-338. https://doi.org/10.1006/brcg.1998.1039

Colavita, F. B. (1974). Human sensory dominance. Perception \& Psychophysics, 16(2), 409-412. https://doi.org/10.3758/BF03203962

Corsi, P. M. (1972). Human memory and the medial temporal region of the brain. McGill University, Montreal, QB.

Desimone, R., \& Duncan, J. (1995). Neural Mechanisms of Selective Visual Attention. Annual Review of Neuroscience, 18, 193-222. https://doi.org/10.1146/annurev-psych-122414-033400

Diaconescu, A. O., Alain, C., \& McIntosh, A. R. (2011). The co-occurrence of multisensory facilitation and cross-modal conflict in the human brain. Journal of Neurophysiology, 106(6), 2896-2909. https://doi.org/10.1152/jn.00303.2011

Diaconescu, A. O., Hasher, L., \& McIntosh, A. R. (2013). Visual dominance and multisensory integration changes with age. NeuroImage, 65, 152-166. https://doi.org/10.1016/j.neuroimage.2012.09.057

Driver, J., \& Spence, C. (1998). Cross-modal links in spatial attention. Philosophical Transactions of the Royal Society B: Biological Sciences, 353(1373), 1319-1331. https://doi.org/10.1098/rstb.1998.0286

Egeth, H. E., \& Sager, L. C. (1977). On the locus of visual dominance. Perception \& Psychophysics, 22(1), 77-86. https://doi.org/10.3758/BF03206083

Gherri, E., \& Eimer, M. (2011). Active listening impairs visual perception and selectivity: An ERP study of auditory dual-task costs on visual attention. Journal of Cognitive Neuroscience, 23(4), 832-844. https://doi.org/10.1162/jocn.2010.21468

Gmeindl, L., \& Courtney, S. M. (2011). Deconstructing Spatial Working Memory and Attention Deficits in Multiple Sclerosis. Neuropsychology, 26(57). https://doi.org/10.1037/a0026213

Green, D. M., \& Swets, J. A. (1974). Signal Detection Theory and Psychophysics. R.E. Krieger Pub. Co.

Hughes, R. W. (2014). Auditory distraction: A duplex-mechanism account. PsyCh Journal, 3(1), 30-41. https://doi.org/10.1002/pchj.44 
Hughes, R. W., Hurlstone, M. J., Marsh, J. E., Jones, D. M., \& Vachon, F. (2013). Cognitive control of auditory distraction: Impact of task difficulty, foreknowledge, and working memory capacity supports duplex-mechanism account. Journal of Experimental Psychology: Human Perception and Performance, 39(2), 539-553. https://doi.org/10.1037/a0029064

Hughes, R. W., \& Marsh, J. E. (2019). When Is Forewarned Forearmed? Predicting Auditory Distraction in Short-Term Memory. Journal of Experimental Psychology: Learning Memory and Cognition, 46(3), 427-442. https://doi.org/10.1037/xlm0000736

Ivry, R. B., \& Spencer, R. M. C. (2004). The neural representation of time. Current Opinion in Neurobiology, 14(2), 225-232. https://doi.org/10.1016/j.conb.2004.03.013

Knudsen, E. I. (2007). Fundamental components of attention. Annual Review of Neuroscience, 30, 57-78. https://doi.org/10.1146/annurev.neuro.30.051606.094256

Lawrence, M. A. (2016). Package 'ez.' In R package 3.1 version (4.4-0). https://github.com/mikelawrence/ez

Lorenc, E. S., Mallett, R., \& Lewis-Peacock, J. A. (2021). Distraction in Visual Working Memory: Resistance is Not Futile. Trends in Cognitive Sciences, 25(3), 228-239. https://doi.org/10.1016/j.tics.2020.12.004

Lukas, S., Philipp, A. M., \& Koch, I. (2010). Switching attention between modalities: Further evidence for visual dominance. Psychological Research, 74(3), 255-267. https://doi.org/10.1007/s00426-0090246-y

Makowski, D. (2018). The psycho package: an efficient and publishing-oriented workflow for psychological science. In Open Source Software (Vol. 3, Issue 22, p. 470).

Marsh, J. E., Campbell, T. A., Vachon, F., Taylor, P. J., \& Hughes, R. W. (2020). How the deployment of visual attention modulates auditory distraction. Attention, Perception, and Psychophysics, 82(1), 350-362. https://doi.org/10.3758/s13414-019-01800-w

Marsh, J. E., Sörqvist, P., \& Hughes, R. W. (2015). Dynamic cognitive control of irrelevant sound: Increased task engagement attenuates semantic auditory distraction. Journal of Experimental Psychology: Human Perception and Performance, 41(5), 1462-1474. https://doi.org/10.1037/xhp0000060

McGurk, H., \& Macdonald, J. (1976). Hearing lips and seeing voices. Nature, 264(5588), 746-748. https://www-naturecom.libproxy1.usc.edu/articles/264746a0.pdf\%0Ahttps://www.nature.com/articles/264746a0.pdf

Mercier, M. R., Foxe, J. J., Fiebelkorn, I. C., Butler, J. S., Schwartz, T. H., \& Molholm, S. (2013). Auditory-driven phase reset in visual cortex : Human electrocorticography reveals mechanisms of early multisensory integration. NeuroImage, 79, 19-29. https://doi.org/10.1016/j.neuroimage.2013.04.060

Meredith, M. A., Nemitz, J. W., \& Stein, B. E. (1987). Determinants of multisensory integration in superior colliculus neurons. I. Temporal factors. The Journal of Neuroscience: The Official Journal of the Society for Neuroscience, 7(10), 3215-3229. https://doi.org/10.1523/jneurosci.07-1003215.1987

Mishra, J., Martinez, A., Sejnowski, T. J., \& Hillyard, S. A. (2007). Early cross-modal interactions in auditory and visual cortex underlie a sound-induced visual illusion. Journal of Neuroscience, 27(15), 4120-4131. https://doi.org/10.1523/JNEUROSCI.4912-06.2007 
Monaco, M., Costa, A., Caltagirone, C., \& Carlesimo, G. A. (2013). Forward and backward span for verbal and visuo-spatial data: Standardization and normative data from an Italian adult population. Neurological Sciences, 34(5), 749-754. https://doi.org/10.1007/s10072-012-1130-x

Myers, N. E., Stokes, M. G., \& Nobre, A. C. (2017). Prioritizing Information during Working Memory: Beyond Sustained Internal Attention. Trends in Cognitive Sciences, 21(6), 449-461. https://doi.org/10.1016/j.tics.2017.03.010

Nobre, A. C., \& van Ede, F. (2018). Anticipated moments: Temporal structure in attention. Nature Reviews Neuroscience, 19(1), 34-48. https://doi.org/10.1038/nrn.2017.141

Peirce, J. W. (2007). PsychoPy-Psychophysics software in Python. Journal of Neuroscience Methods, 162(1-2), 8-13. https://doi.org/10.1016/j.jneumeth.2006.11.017

R Core Team. (2018). R: A Language and Environment for Statistical Computing. https://www.rproject.org/

Rudis, B. (2018). Hrbrthemes: Additional themes, theme components and utilities for "ggplot2." In Hrbrthemes Documentation.

Schröger, E., \& Widmann, A. (1998). Speeded responses to audiovisual signal changes result from bimodal integration. Psychophysiology, 35(6), 755-759. https://doi.org/10.1017/S0048577298980714

Shams, L., Kamitani, Y., \& Shimojo, S. (2000). Illusions. What you see is what you hear. Nature, 408(December), 788.

Soto-Faraco, S., Kingstone, A., \& Spence, C. (2004). Cross-Modal Dynamic Capture: Congruency Effects in the Perception of Motion Across Sensory Modalities. Journal of Experimental Psychology: Human Perception and Performance, 30(2), 330-345. https://doi.org/10.1037/00961523.30.2.330

Stein, B. E., Meredith, M. A., Huneycutt, W. S., \& McDade, L. (1989). Behavioral indices of multisensory integration: Orientation to visual cues is affected by auditory stimuli. Journal of Cognitive Neuroscience, 1(1), 12-24. https://doi.org/10.1162/jocn.1989.1.1.12

Stein, Barry E., London, N., Wilkinson, L. K., \& Price, D. D. (1996). Enhancement of perceived visual intensity by auditory stimuli: A psychophysical analysis. Journal of Cognitive Neuroscience, $8(6)$, 497-506. https://doi.org/10.1162/jocn.1996.8.6.497

Talsma, D., Senkowski, D., Soto-Faraco, S., \& Woldorff, M. G. (2010). The multifaceted interplay between attention and multisensory integration. Trends in Cognitive Sciences, 14(9), 400-410. https://doi.org/10.1016/j.tics.2010.06.008

Theeuwes, J. (2014). Spatial orienting and attentional capture. In A. C. Nobre \& S. Kastner (Eds.), The Oxford Handbook of Attention (pp. 231-252). Oxford University Press Oxford.

Todorovic, A., van Ede, F., Maris, E., \& de Lange, F. P. (2011). Prior expectation mediates neural adaptation to repeated sounds in the auditory cortex: An MEG study. Journal of Neuroscience, 31(25), 9118-9123. https://doi.org/10.1523/JNEUROSCI.1425-11.2011

Treisman, A. M. (1964). Selective attention in man. British Medical Bulletin, 20(1), 12-16. https://doi.org/10.1093/oxfordjournals.bmb.a070274

Van der Burg, E., Olivers, C. N. L., Bronkhorst, A. W., \& Theeuwes, J. (2008). Pip and Pop: Nonspatial Auditory Signals Improve Spatial Visual Search. Journal of Experimental Psychology: Human 
Perception and Performance, 34(5), 1053-1065. https://doi.org/10.1037/0096-1523.34.5.1053

van Ede, F., \& Nobre, A. C. (2021). Toward a neurobiology of internal selective attention. Trends in Neurosciences, $x x(\mathrm{xx}), 1-3$. https://doi.org/10.1016/j.tins.2021.04.010

Villena-González, M., López, V., \& Rodríguez, E. (2016). Orienting attention to visual or verbal/auditory imagery differentially impairs the processing of visual stimuli. NeuroImage, 132, 71-78. https://doi.org/10.1016/j.neuroimage.2016.02.013

Vroomen, J., \& De Gelder, B. (2000). Sound enhances visual perception: Cross-modal effects of auditory organization on vision. Journal of Experimental Psychology: Human Perception and Performance, 26(5), 1583-1590. https://doi.org/10.1037/0096-1523.26.5.1583

Welch, R. B. (1999). Meaning, attention, and the "“unity assumption"” in the intersensory bias ofspatial and temporal perceptions. In G. Aschersleben, T. Bachmann, \& J. Müsseler (Eds.), Cognitive Contributions to the Perception of Spatial and Temporal Events. Elsevier Science B.V.

Welch, R. B., \& Warren, D. H. (1980). Immediate perceptual response to intersensory discrepancy. Psychological Bulletin, 88(3), 638-667. https://doi.org/10.1037/0033-2909.88.3.638

Wetzel, N., Buttelmann, D., Schieler, A., \& Widmann, A. (2016). Infant and adult pupil dilation in response to unexpected sounds. Developmental Psychobiology, 58(3), 382-392. https://doi.org/10.1002/dev.21377

Wickham, H. (2016). ggplot2: Elegant Graphics for Data Analysis. Springer-Verlag New York. https://ggplot2.tidyverse.org

Wickham, H., François, R., Henry, L., \& Müller, K. (2021). dplyr: A Grammar of Data Manipulation (1.0.7). https://github.com/tidyverse/dplyr

Wilke, C. O., Wickham, H., \& Wilke, M. C. O. (2019). Package 'cowplot.' In Streamlined Plot Theme and Plot Annotations for 'ggplot2. 